\title{
A cross-sectional study of irritable bowel syndrome in nurses in China: prevalence and associated psychological and lifestyle factors*
}

\author{
Liang LIU $^{1,2}$, Qi-fan XIAO ${ }^{1,3}$, Yan-li ZHANG ${ }^{\dagger+3}$, Shu-kun YAO Y1,2 $^{\dagger 11,2}$ \\ $\left({ }^{1}\right.$ Department of Gastroenterology, China-Japan Friendship Hospital, Beijing 100029, China) \\ $\left({ }^{2}\right.$ Graduate School, Peking Union Medical College \& Chinese Academy of Medical Sciences, Beijing 100730, China) \\ ( ${ }^{3}$ Peking University Health Science Center, Beijing 100151, China) \\ †E-mail: janetzhyl@gmail.com; ysk12329@126.com \\ Received June 14, 2013; Revision accepted Oct. 12, 2013; Crosschecked May 25, 2014
}

\begin{abstract}
The prevalence of irritable bowel syndrome (IBS) and associated factors, especially psychological and lifestyle factors, in nurses in China have not been investigated previously. The aims of this study were to assess the prevalence of IBS in nurses, to evaluate whether factors, such as psychological disorders, are associated with IBS, and to determine whether psychological disorders can influence the severity of symptoms of IBS and quality of life (QOL). A cross-sectional study was conducted for Chinese nurses from November 2012 to February 2013. Participants were asked to complete questionnaires. The prevalence of IBS was $17.4 \%$. The revised symptom checklist 90 (SCL-90-R) scores were significantly higher for nurses with IBS than for those without IBS $(P<0.001)$, and no difference in scores between IBS subtypes was found $(F=1.893, P=0.142)$. The scores of $Q O L$ for nurses with and without IBS were $77.18 \pm 21.93$ and $88.44 \pm 11.89(P<0.001)$, respectively. Psychological disorders did not show statistically significant correlations with severity of symptoms of IBS or QOL. Alcohol consumption, low level of exercise, and psychological disorders were risk factors for IBS. In summary, nurses in China show a high prevalence of IBS. Psychological disorders and some related lifestyle factors are probably responsible for the development of IBS in nurses.
\end{abstract}

Key words: Irritable bowel syndrome (IBS), Prevalence, Psychosocial disorder, Quality of life (QOL) doi: $10.1631 /$ jzus.B1300159

Document code: A

CLC number: R574.4

\section{Introduction}

Irritable bowel syndrome (IBS) is one of the most common functional bowel disorders, defined as the presence of abdominal pain or discomfort in association with altered bowel habits, without any organic damages to the intestine (Fan et al., 2006). In most western countries, the prevalence of IBS is about 20\%, while, in Asia, the prevalence varies from

\footnotetext{
$\ddagger$ Corresponding authors

* Project supported by the National Natural Science Foundation of China (No. 81070299)

(C) Zhejiang University and Springer-Verlag Berlin Heidelberg 2014
}

$2.9 \%$ to $15.6 \%$ (Chang and $\mathrm{Lu}, 2007$; Gwee et al., 2010). IBS has been reported to account for up to $25 \%$ of the outpatient workload of a gastroenterologist (Butt et al., 2012), and causes the significant direct and indirect costs related to medical investment, impaired work productivity, and quality of life (QOL).

The pathophysiology of IBS is still not completely understood, but psychological disorders may affect the onset and outcome of IBS in some patients. Studies have revealed that a much higher percentage $(40 \%-60 \%)$ of IBS patients experience underlying psychological disorders than in the control groups without IBS (Butt et al., 2012). Anxiety, depression, and somatization are more prevalent in IBS patients. 
Jerndal et al. (2010) found that anxiety, which is an important mediating factor, greatly affects the severity of symptoms and QOL, and that reduction of anxiety can achieve a general improvement in IBS symptoms. Two recent studies concluded that depression and somatization were independently associated with IBS (Nicholl et al., 2008; Savas et al., 2009).

In recent years, the number of IBS research papers, relating to specific groups of people, has increased in China and the rest of the world. A survey of college and university students in North China found that IBS was common, and psychological disorders posed significant risks for IBS (Dong et al., 2010). One study in the USA found that nurses, especially those working on rotating shifts, were at a high risk for developing IBS (Nojkov et al., 2010). Zhen et al. (2006) also demonstrated that IBS was common and severe among nurses working on rotating shifts, and sleep disturbance might be associated with IBS symptoms.

To our knowledge, the prevalence of IBS and associated factors, especially psychological factors, has not been investigated for nurses in China. The aims of this study were:

(1) To assess the prevalence of IBS in nurses;

(2) To evaluate whether factors, such as psychological disorders, are associated with IBS;

(3) To determine whether psychological disorders can influence the severity of symptoms of IBS and QOL.

\section{Materials and methods}

\subsection{Study setting}

This cross-sectional study was carried out from Nov. 2012 to Feb. 2013 in the China-Japan Friendship Hospital, Beijing, China. All nurses (450) employed in the clinical departments were invited to voluntarily complete questionnaires. The participants gave their written informed consent prior to data collection. The study was approved by the Ethics Committee of the China-Japan Friendship Hospital, Beijing, China.

\subsection{Criteria of exclusion}

Subjects with self-reported organic gastrointestinal disorder, abnormal laboratory findings, weight loss, anemia, bloody stools, family history of cancer, and other alarming signs, and those who were currently pregnant or had previously undergone gastrointestinal surgery were excluded from the study.

\subsection{Criteria for IBS patients}

All subjects who were positive for IBS, using Rome III criteria, were categorized as IBS patients, while those who were found negative for IBS were categorized as controls.

\subsection{Lifestyle factors}

Questions about lifestyles, such as alcohol consumption, smoking, physical exercise, and night-shift work, were included in the questionnaire. In the assessment of the frequency of physical exercise in the questionnaire, "often" means the frequency of exercise at a minimum of three times a week with a minimum of $30 \mathrm{~min}$ for each session.

\subsection{Questionnaires}

\subsubsection{Chinese version of Rome III questionnaire}

Rome III criteria were established by the Rome III Committee in 2006 (Longstreth et al., 2006). The Chinese version has been widely used in China in recent years (Dong et al., 2013). The diagnosis of IBS was based on the presence of abdominal pain or discomfort for at least three months during the previous six months, with at least two or more of the following conditions: symptoms associated with a change in frequency or form of stool, pain improved after defecation. Patients with IBS were divided into diarrhea-predominant IBS (IBS-D), constipationpredominant IBS (IBS-C), mixed IBS (IBS-M), and un-subtyped IBS (IBS-U) according to the proportion of hard and lumpy stools (Dong et al., 2013).

\subsection{2 'Severity of IBS' questionnaire}

The IBS severity questionnaire includes five items, which were assessed using the irritable bowel severity scoring system based on the presence and severity of perceived pain, the frequency of abdominal pain or discomfort, the presence and severity of abdominal distension, satisfaction with bowel habits, and the quantification of interference in the patient's general lifestyle by these symptoms. Each item uses a 
100 -point scale and the final severity score is the sum of these scores (maximum up to 500). Patients were classified as having mild IBS (IBS severity score 75-175), moderately severe IBS (score 175-300), or severe IBS (score > 300) (Francis et al., 1997; Singh et al., 2012).

\subsubsection{Revised symptom checklist 90 (SCL-90-R)} questionnaire

Psychopathological features were assessed with the SCL-90-R questionnaire, which contains 90 questions, on nine different aspects: somatization, depression, anxiety, obsessive-compulsive, interpersonal sensitivity, anger-hostility, phobic anxiety, paranoid ideation, and psychoticism. The general symptom index (GSI), which represents the average severity score of all 90 items on the questionnaire, is considered to be a reliable measure of psychological distress (Schauenburg and Strack, 1998; 1999; Piacentino et al., 2011).

\subsubsection{Health-related QOL questionnaire}

The disease-specific QOL for IBS (IBS-QOL) is a 34-item self-report questionnaire, which was designed to evaluate QOL in IBS patients. The questionnaire includes eight subscales, each item having a five-point response scale. The total score of the QOL $(0-100)$ is a general parameter reflecting the health status of IBS patients. Higher total scores indicated better QOL (Dai et al., 2008).

\subsection{Statistical analysis}

Statistical analysis was performed using the SPSS version 18.0.

Distributions of demographic characteristics and lifestyle factors were analyzed by Pearson's $\chi^{2}$ or Fisher's exact test. The $t$-test was used to compare the SCL-90-R scores between IBS and the control groups. Comparisons between different IBS subgroups were performed using the analysis of variance (ANOVA) test for continuous data. Spearman's correlations between QOL and IBS severity, between SCL-90-R and IBS severity, and between QOL and SCL-90-R among IBS patients were determined. Logistic regression analysis was used to assess the possible risk factors. The odds ratio (OR) with a $95 \%$ confidence interval $(\mathrm{CI})$ was calculated. Data are presented as mean \pm standard deviation (SD). $P<0.05$ was considered statistically significant.

\section{Results}

\subsection{Response rate, demographic characteristics, and lifestyle factors of participants}

Of the 450 nurses, $346(76.9 \%)$ completed the surveys. Following the exclusion criteria, 340 nurses were included in the final analyses. Of the 340 nurses, $11(3.2 \%)$ were men and $329(96.8 \%)$ were women, with a mean age of $(31.47 \pm 7.59)$ years. The demographic characteristics and lifestyle factors of participants are listed in Tables 1 and 2. No difference in the demographic characteristics between IBS patients and the control group was found; however, there were statistically significant differences in lifestyle factors (night shift, alcohol consumption, and physical exercise) between the two groups.

\subsection{Prevalence of IBS and its subtypes}

Of the 340 nurses, 59 (all women) fulfilled the criteria for having IBS. The prevalence of IBS was $17.4 \%, 9$ cases were IBS-C (15.3\%), 14 cases were IBS-D (23.7\%), 23 cases were IBS-M (39.0\%), and 13 cases were IBS-U (22.0\%).

\subsection{SCL-90-R scores in IBS patients and controls}

IBS patients showed a significantly higher mean score for the GSI and nine subscale scores compared with the control group $(P<0.001$; Table 3$)$. The GSI scores for the IBS-C, IBS-D, IBS-M, and IBS-U groups were $0.76 \pm 0.47,0.71 \pm 0.45,1.20 \pm 1.00$, and $0.66 \pm 0.77$, respectively. No statistically significant difference was found between IBS subtypes ( $F=1.893$, $P=0.142$ ).

\subsection{QOL scores in IBS patients and controls}

The QOL score for IBS patients was 77.18 21.93, and it was $88.44 \pm 11.89(P<0.001)$ for those without IBS. This indicated that the occurrence of IBS can influence their daily health and QOL. No difference was found between IBS subtypes ( $F=0.581$, $P=0.805$ ). 
Table 1 Demographic characteristics of nurses

\begin{tabular}{|c|c|c|c|c|}
\hline Nurse & $\begin{array}{c}\mathrm{IBS}^{*} \\
(n=59)\end{array}$ & $\begin{array}{l}\text { Control }^{*} \\
(n=281)\end{array}$ & $\begin{array}{c}\text { Total } \\
(n=340)\end{array}$ & $P$ \\
\hline \multicolumn{5}{|l|}{$\overline{\operatorname{Sex}}$} \\
\hline Male & $0(0 \%)$ & $11(100 \%)$ & 11 & 0.122 \\
\hline Female & $59(17.9 \%)$ & $270(82.1 \%)$ & 329 & \\
\hline \multicolumn{5}{|l|}{ Age (year) } \\
\hline $20-29$ & $32(18.1 \%)$ & $145(81.9 \%)$ & 177 & 0.882 \\
\hline $30-39$ & $19(17.9 \%)$ & $87(82.1 \%)$ & 106 & \\
\hline $40-49$ & $7(13.5 \%)$ & $45(86.5 \%)$ & 52 & \\
\hline $50-59$ & $1(20.0 \%)$ & $4(80.0 \%)$ & 5 & \\
\hline \multicolumn{5}{|l|}{ Nationality } \\
\hline Han & $56(17.5 \%)$ & $264(82.5 \%)$ & 320 & 0.775 \\
\hline $\begin{array}{l}\text { Minority } \\
\text { groups }\end{array}$ & $3(15.0 \%)$ & $17(85.0 \%)$ & 20 & \\
\hline \multicolumn{5}{|l|}{ Marital status } \\
\hline Unmarried & $25(18.4 \%)$ & $111(81.6 \%)$ & 136 & 0.348 \\
\hline Married & $32(16.1 \%)$ & $167(83.9 \%)$ & 199 & \\
\hline Divorce & $2(40.0 \%)$ & $3(60.0 \%)$ & 5 & \\
\hline \multicolumn{5}{|l|}{ Level of education } \\
\hline $\begin{array}{l}\text { Secondary } \\
\text { school }\end{array}$ & $5(26.3 \%)$ & $14(73.7 \%)$ & 19 & 0.230 \\
\hline Junior college & $22(15.5 \%)$ & $120(84.5 \%)$ & 142 & \\
\hline Undergraduate & $32(19.2 \%)$ & $135(80.8 \%)$ & 167 & \\
\hline Master degree & $0(0 \%)$ & $12(100 \%)$ & 12 & \\
\hline
\end{tabular}

* Data are expressed as number (percentage)

Table 2 Lifestyle factors in IBS patients and controls

\begin{tabular}{ccrrr}
\hline $\begin{array}{c}\text { Lifestyle } \\
\text { factor }\end{array}$ & $\begin{array}{c}\text { IBS }^{*} \\
(n=59)\end{array}$ & \multicolumn{1}{c}{$\begin{array}{c}\text { Control }^{*} \\
(n=281)\end{array}$} & $\begin{array}{c}\text { Total } \\
(n=340)\end{array}$ & $P$ \\
\hline $\begin{array}{c}\text { Night shift } \\
>5 \mathrm{~d} / \text { time }\end{array}$ & $7(9.6 \%)$ & $66(90.4 \%)$ & 73 & 0.048 \\
$\leq 5 \mathrm{~d} /$ time & $52(19.5 \%)$ & $215(80.5 \%)$ & 267 & \\
Smoking & & & & \\
No & $57(17.2 \%)$ & $274(82.8 \%)$ & 331 & 0.696 \\
Yes & $2(22.2 \%)$ & $7(77.8 \%)$ & 9 &
\end{tabular}

Alcohol consumption

$\begin{array}{llrrr}\text { No } & 41(14.6 \%) & 240(85.4 \%) & 281 & 0.003 \\ \text { Yes } & 18(30.5 \%) & 41(69.5 \%) & 59 & \end{array}$

Physical exercise

$\begin{array}{lcccc}\text { Seldom } & 51(24.5 \%) & 157(75.5 \%) & 208 & <0.001 \\ \text { Often } & 8(6.1 \%) & 124(93.9 \%) & 132 & \end{array}$

* Data are expressed as number (percentage)
Table 3 Comparison of SCL-90-R scores between IBS patients and controls

\begin{tabular}{lcc}
\hline \multirow{2}{*}{\multicolumn{1}{c}{ Factor }} & \multicolumn{2}{c}{ SCL-90-R score } \\
\cline { 2 - 3 } & $\begin{array}{c}\text { IBS } \\
(n=59)\end{array}$ & $\begin{array}{c}\text { Control } \\
(n=281)\end{array}$ \\
\hline Somatization & $1.05 \pm 0.78^{*}$ & $0.46 \pm 0.46$ \\
Obsessive-compulsive & $1.16 \pm 0.84^{*}$ & $0.62 \pm 0.62$ \\
Interpersonal sensitivity & $0.89 \pm 0.85^{*}$ & $0.42 \pm 0.52$ \\
Depression & $0.94 \pm 0.86^{*}$ & $0.45 \pm 0.53$ \\
Anxiety & $0.90 \pm 0.89^{*}$ & $0.43 \pm 0.50$ \\
Hostility & $1.03 \pm 0.98^{*}$ & $0.44 \pm 0.55$ \\
Phobic anxiety & $0.57 \pm 0.79^{*}$ & $0.25 \pm 0.41$ \\
Paranoid ideation & $0.69 \pm 0.86^{*}$ & $0.33 \pm 0.45$ \\
Psychoticism & $0.69 \pm 0.85^{*}$ & $0.30 \pm 0.43$ \\
GSI & $0.90 \pm 0.80^{*}$ & $0.42 \pm 0.45$ \\
\hline
\end{tabular}

GSI: general symptom index. ${ }^{*} P<0.001$ vs. control. Data are presented as mean $\pm \mathrm{SD}$

\subsection{Distribution of IBS subtypes in IBS severity}

Of the 59 IBS patients, 15 had mild IBS, 44 had moderately severe IBS, and none had severe IBS (Table 4). Thus IBS was moderately severe in the majority of the cases.

Table 4 Distribution of IBS subtypes in IBS severity

\begin{tabular}{cccc}
\hline IBS & \multicolumn{3}{c}{ IBS severity } \\
\cline { 2 - 4 } subtype & Mild & Moderate & Severe \\
\hline IBS-C & 0 & 9 & 0 \\
IBS-D & 3 & 11 & 0 \\
IBS-M & 6 & 17 & 0 \\
IBS-U & 6 & 7 & 0 \\
\hline
\end{tabular}

IBS-C: constipation-predominant IBS; IBS-D: diarrhea-predominant IBS; IBS-M: mixed IBS; IBS-U: un-subtyped IBS

\subsection{Spearman's correlation coefficients for IBS patients}

Spearman's correlations between SCL-90-R and IBS severity, SCL-90-R and QOL, QOL and IBS severity were analyzed. No significant correlations were found between them (Table 5). 
Table 5 Spearman's correlation coefficients for IBS patients

\begin{tabular}{lrc}
\hline \multicolumn{1}{c}{ Type } & $\begin{array}{c}\text { Correlation } \\
\text { coefficient }\end{array}$ & $P$ \\
& 0.196 & 0.137 \\
SCL-90-R score (GSI) and IBS severity & -0.058 & 0.662 \\
SCL-90-R score (GSI) and QOL & 0.177 & 0.180 \\
QOL and IBS severity & &
\end{tabular}

GSI: general symptom index; QOL: quality of life

\subsection{Risk factors for IBS}

After the univariate analysis, to find independent risk factors for IBS, we entered factors (night shift, alcohol consumption, physical exercise, SCL-90-R score (GSI)) into a multivariable logistic regression analysis. The results showed that alcohol consumption $(P=0.010)$, low exercise level $(P<0.001)$, and a SCL-90-R score (GSI) $(P<0.001)$ were independently associated with IBS (Table 6).

Table 6 Evaluation of risk factors for IBS by multivariate logistic regression analysis

\begin{tabular}{|c|c|c|c|c|c|}
\hline Risk factor & $\beta$ & SE & Wals & $\begin{array}{c}\text { Odds ratio } \\
(95 \% \mathrm{CI})\end{array}$ & $P$ \\
\hline Night shift & 0.503 & 0.461 & 1.192 & $\begin{array}{c}0.605 \\
(0.245-1.492)\end{array}$ & 0.275 \\
\hline $\begin{array}{l}\text { Alcohol } \\
\text { consumption }\end{array}$ & 0.959 & 0.374 & 6.564 & $\begin{array}{c}0.383 \\
(0.184-0.798)\end{array}$ & 0.010 \\
\hline $\begin{array}{l}\text { Physical } \\
\text { exercise }\end{array}$ & 1.620 & 0.416 & 15.199 & $\begin{array}{c}5.054 \\
(2.238-11.414)\end{array}$ & $<0.001$ \\
\hline $\begin{array}{l}\text { SCL-90-R } \\
\text { score (GSI) }\end{array}$ & 1.230 & 0.284 & 18.738 & $\begin{array}{c}3.421 \\
(1.960-5.971)\end{array}$ & $<0.001$ \\
\hline
\end{tabular}

SE: standard error; CI: confidence interval; GSI: general symptom index

\section{Discussion}

To our knowledge, this was the first investigation using Rome III criteria to assess the prevalence and the associated psychological and lifestyle factors of IBS in nurses in China. The prevalence of IBS in the study was $17.4 \%$, and $39 \%$ of those with IBS had IBS-M. The SCL-90-R scores were significantly higher for IBS patients than for the control group.

It is likely that changing lifestyles and the rapid changes in the socioeconomic environment contribute to the increased prevalence of IBS in Asian countries. Different eating habits, genetic factors, and sociocultural backgrounds may result in different prevalence rates, based on investigations conducted from different perspectives in China. A population-based study estimated the prevalence of IBS as 7\% in Guangdong, China (Chang and Lu, 2007). The prevalence of IBS among undergraduates in Southeast China was reported as $10.4 \%$ using Rome III criteria (Dai et al., 2008), and similarly, Dong et al. (2010) reported the prevalence of IBS in college and university students in North China as $7.85 \%$. Our survey suggests that nurses have an even higher prevalence of IBS. Nojkov et al. (2010) found similar results in nurses in the USA, in which the prevalence of IBS was $48 \%$ in rotating-shift nurses and $31 \%$ in day-shift nurses. A study from Singapore found the prevalence of functional bowel disorders to be $38 \%$ in nurses working rotating shifts and $20 \%$ in those working day shifts (Zhen et al., 2006). Using Rome III criteria and only nurses being included may explain this higher prevalence. Moreover, because of their particular working conditions (experiencing more of the various psychosocial stressors, heavy workloads, and lack of rest time), nurses may be an especially high-risk population for IBS.

In our survey, the SCL-90-R scores were high in nurses with IBS, indicating that psychosocial disorders have some influence on the clinical course of IBS. Psychiatric symptoms and psychiatric disorders are common among IBS patients, and the most frequent psychiatric diagnoses in IBS are depression, anxiety, and somatoform disorders (Surdea-Blaga et al., 2012). Savas et al. (2009) reported that the prevalence of depression in IBS patients was $31.4 \%$ compared with $17.5 \%$ in the control group. In a community study, anxiety was found to be more common in IBS patients (Lee et al., 2009). Sykes et al. (2003) observed that anxiety tends to precede IBS onset and plays an important role in the development of IBS. In a South Australian study, Mikocka-Walus et al. (2008) found that depression and anxiety were common in subjects with IBS symptoms. An excessive tendency to somatization was reported in population-based studies and in clinical studies (Locke et al., 2004). Somatization, together with depression and anxiety, may 
explain the extra-gastrointestinal symptoms in IBS patients, such as headaches, constant fatigue, and urinary symptoms (Zimmerman, 2003). Increasing attention has been given to the influence of psychosocial factors in the pathogenesis, severity, course, and outcome of IBS. The bi-directional connection between the gastrointestinal tract and the brain is through the brain-gut axis, and psychosocial factors can lead to dysfunction of the brain-gut axis, which in turn causes dysfunction of the gut (Jones et al., 2006).

In the present study, the findings suggest that the nurses with low exercise levels were more likely to develop IBS, which is consistent with Dong et al. (2010). The chronic abdominal discomfort or pain may reduce the motivation of nurses to exercise, or they may be too busy to do it (Dong et al., 2010). Our survey indicates that alcohol consumption is a risk factor for IBS patients. We speculate that alcohol can increase intestinal membrane permeability and visceral sensitivity, thus changing the normal physiological function of the gut. Our findings that there were no statistically significant differences in QOL and SCL-90-R scores between IBS subtypes are consistent with the results of other previous research (Katsinelos et al., 2009; Park et al., 2009; Jamali et al., 2012). However, Eriksson et al. (2008) found that QOL was lower in IBS-C and IBS-M when compared with other subtypes, and showed that the mean psychosocial scores were higher in IBS-M and IBS-C compared with IBS-D. This can be explained by the different types of questionnaires, cultures, and selected subjects. In our study, IBS was moderately severe in the majority of cases, and no statistically significant correlations between SCL-90-R and IBS severity, between SCL-90-R and QOL, or between QOL and IBS severity were found. This indicated that, in nurses with IBS, psychosocial factors were not associated with QOL or severity of symptoms, and that severity of symptoms did not affect QOL. If we followed the nurses with IBS for long enough periods, however, IBS subtypes may change into one another, and comparisons of these parameters might change (Mearin et al., 2004).

A number of limitations of our study should be taken into consideration. First, the survey was restricted to nurses, which might influence the genera- lizability of our results. Second, the study was based on self-reporting questionnaires, without using upper gastrointestinal endoscopy and colonoscopy to exclude structural intestinal diseases. Third, there were only 11 men in our study, so the findings may not be generally applicable to male nurses.

\section{Conclusions}

In summary, this study showed that the prevalence of IBS in nurses is $17.4 \%$. Psychological disorders and certain related lifestyle factors are probably the elements that make nurses particularly susceptible to the development of IBS. Further studies should pay more attention to psychiatric evaluation and treatment together with rectification of some unhealthy aspects of current and past lifestyles.

\section{Compliance with ethics guidelines}

Liang LIU, Qi-fan XIAO, Yan-li ZHANG, and Shu-kun YAO declare that they have no conflict of interest.

All procedures followed were in accordance with the ethical standards of the responsible committee on human experimentation (institutional and national) and with the Helsinki Declaration of 1975, as revised in 2008 (5). Informed consent was obtained from all patients for being included in the study.

\section{References}

Butt, A.S., Salih, M., Jafri, W., et al., 2012. Irritable bowel syndrome and psychiatric disorders in Pakistan: a case control study. Gastroenterol. Res. Pract., 2012:291452. [doi:10.1155/2012/291452]

Chang, F.Y., Lu, C.L., 2007. Irritable bowel syndrome in the 21st century: perspectives from Asia or South-east Asia. $J$. Gastroenterol. Hepatol., 22(1):4-12. [doi:10.1111/j.14401746.2006.04672.x]

Dai, N., Cong, Y., Yuan, H., 2008. Prevalence of irritable bowel syndrome among undergraduates in Southeast China. Dig. Liver Dis., 40(6):418-424. [doi:10.1016/j. dld.2008.01.019]

Dong, Y.Y., Zuo, X.L., Li, C.Q., et al., 2010. Prevalence of irritable bowel syndrome in Chinese college and university students assessed using Rome III criteria. World J. Gastroenterol., 16(33):4221-4226. 
Dong, Y.Y., Chen, F.X., Yu, Y.B., et al., 2013. A school-based study with Rome III criteria on the prevalence of functional gastrointestinal disorders in Chinese college and university students. PLOS ONE, 8(1):e54183. [doi:10. 1371/journal.pone.0054183]

Eriksson, E.M., Andren, K.I., Eriksson, H.T., et al., 2008. Irritable bowel syndrome subtypes differ in body awareness, psychological symptoms and biochemical stress markers. World J. Gastroenterol., 14(31):4889-4896.

Fan, Y.J., Chen, S.J., Yu, Y.C., et al., 2006. A probiotic treatment containing Lactobacillus, Bifidobacterium and Enterococcus improves IBS symptoms in an open label trial. J. Zhejiang Univ.-Sci. B (Biomed. \& Biotechnol.), 7(12):987-991. [doi:10.1631/jzus.2006.B0987]

Francis, C.Y., Morris, J., Whorwell, P.J., 1997. The irritable bowel severity scoring system: a simple method of monitoring irritable bowel syndrome and its progress. Aliment Pharmacol. Ther., 11(2):395-402. [doi:10.1046/j.13652036.1997.142318000.x]

Gwee, K.A., Bak, Y.T., Ghoshal, U.C., et al., 2010. Asian consensus on irritable bowel syndrome. J. Gastroenterol. Hepatol., 25(7):1189-1205. [doi:10.1111/j.1440-1746. 2010.06353.x]

Jamali, R., Jamali, A., Poorrahnama, M., et al., 2012. Evaluation of health related quality of life in irritable bowel syndrome patients. Health Qual. Life Outcomes, 10(1):12 [doi:10.1186/1477-7525-10-12]

Jerndal, P., Rinqström, G., Agerforz, P., et al., 2010. Gastrointestinal-specific anxiety: an important factor for severity of GI symptoms and quality of life in IBS. Neurogastroenterol. Motil., 22(6):646-e179. [doi:10.1111/j. 1365-2982.2010.01493.x]

Jones, M.P., Dilley, J.B., Drossman, D., et al., 2006. Brain-gut connections in functional GI disorders: anatomic and physiologic relationships. Neurogastroenterol. Motil., 18(2): 91-103. [doi:10.1111/j.1365-2982.2005.00730.x]

Katsinelos, P., Lazaraki, G., Kountouras, J., et al., 2009. Prevalence, bowel habit subtypes and medical care-seeking behaviour of patients with irritable bowel syndrome in Northern Greece. Eur. J. Gastroenterol. Hepatol., 21(2): 183-189. [doi:10.1097/MEG.0b013e328312eb97]

Lee, S., Wu, J., Ma, Y.L., et al., 2009. Irritable bowel syndrome is strongly associated with generalized anxiety disorder: a community study. Aliment. Pharmacol. Ther., 30(6): 643-651. [doi:10.1111/j.1365-2036.2009.04074.x]

Locke, G.R., Weaver, A.L., Melton, L.J., et al., 2004. Psychosocial factors are linked to functional gastrointestinal disorders: a population based nested case-control study. Am. J. Gastroenterol., 99(2):350-357. [doi:10.1111/j. 1572-0241.2004.04043.x]
Longstreth, G.F., Thompson, W.G., Chey, W.D., et al., 2006. Functonal bowel disorders. Gastroenterology, 130(5): 1480-1491. [doi:10.1053/j.gastro.2005.11.061]

Mearin, F., Baro, E., Roset, M., et al., 2004. Clinical patterns over time in irritable bowel syndrome: symptom instability and severity variability. Am. J. Gastroenterol., 99(1): 113-121. [doi:10.1046/j.1572-0241.2003.04023.x]

Mikocka-Walus, A.A., Turnbull, D.A., Andrews, J.M., et al., 2008. Psychological problems in gastroenterology outpatients: a South Australian experience. Psychological co-morbidity in IBD, IBS and hepatitis C. Clin. Pract. Epidemiol. Ment. Health, 4(1):15. [doi:10.1186/17450179-4-15]

Nicholl, B.I., Halder, S.L., Macfarlane, G.J., et al., 2008. Psychosocial risk markers for new onset irritable bowel syndrome-results of a large prospective populationbased study. Pain, 137(1):147-155. [doi:10.1016/j.pain. 2007.08.029]

Nojkov, B., Rubenstein, J.H., Chey, W.D., et al., 2010. The impact of rotating shift work on the prevalence of irritable bowel syndrome in nurses. Am. J. Gastroenterol., 105(4): 842-847. [doi:10.1038/ajg.2010.48]

Park, J.M., Choi, M.G., Kim, Y.S., et al., 2009. Quality of life of patients with irritable bowel syndrome in Korea. Qual. Life Res., 18(4):435-446. [doi:10.1007/s11136-009-9461-7]

Piacentino, D., Cantarini, R., Alfonsi, M., et al., 2011. Psychopathological features of irritable bowel syndrome patients with and without functionaldyspepsia: a cross sectional study. BMC Gastroenterol., 11(1):94. [doi:10.1186/1471230X-11-94]

Savas, L.S., White, D.L., Wieman, M., et al., 2009. Irritable bowel syndrome and dyspepsia among women veterans: prevalence and association with psychological distress. Aliment. Pharmacol. Ther., 29(1):115-125. [doi:10.1111/j. 1365-2036.2008.03847.x]

Schauenburg, H., Strack, M., 1998. The symptom checklist90-R (SCL-90-R) for presenting statistically and clinically significant psychotherapy outcome. Psychother. Psychosom. Med. Psychol., 48(7):257-264.

Schauenburg, H., Strack, M., 1999. Measuring psychotherapeutic change with the symptom checklist SCL $90 \mathrm{R}$. Psychother. Psychosom., 68(4):199-206. [doi:10.1159/ 000012333]

Singh, P., Agnihotri, A., Pathak, M.K., et al., 2012. Psychiatric, somatic and other functional gastrointestinal disorders in patients with irritable bowel syndrome at a tertiary care center. J. Neurogastroenterol. Motil., 18(3):324-331. [doi:10.5056/jnm.2012.18.3.324]

Surdea-Blaga, T., Băban, A., Dumitrascu, D.L., 2012. Psychosocial determinants of irritable bowel syndrome. World J. Gastroenterol., 18(7):616-626. [doi:10.3748/ wjg.v18.i7.616] 
Sykes, M.A., Blanchard, E.B., Lackner, J., et al., 2003. Psychopathology in irritable bowel syndrome: support for a psychophysiological model. J. Behav. Med., 26(4): 361-372. [doi:10.1023/A:1024209111909]

Zhen, L.W., Ann, G.K., Yu, H.K., 2006. Functional bowel disorders in rotating shift nurses may be related to sleep disturbances. Eur. J. Gastroenterol. Hepatol., 18(6):623-627.
Zimmerman, J., 2003. Extraintestinal symptoms in irritable bowel syndrome and inflammatory bowel diseases: nature, severity, and relationship to gastrointestinal symptoms. Dig. Dis. Sci., 48(4):743-749. [doi:10.1023/A: 1022840910283]

\section{中文嫩要：}

本文题目: 护士人群肠易激综合征患病情况及其可能致病因素的横断面研究

A cross-sectional study of irritable bowel syndrome in nurses in China: prevalence and associated psychological and lifestyle factors

研究目的: 调查护士肠易激综合征 (IBS) 的患病率以及精神心理因素等是否为 IBS 患病的危险因素, 明 确精神心理因素是否影响到 IBS 患者的症状严重性和生活质量。

创新要点: 在中国, 针对特殊人群（如护士）IBS 患病率及其影响因素的调查相对较少。特殊人群因工 作性质等原因, 可能成为 IBS 的高发人群, 了解其患病情况和可能的致病因素为针对性治疗 提供理论依据。

研究方法：针对北京某三甲医院临床科室护士采取自愿填写封闭式问卷的方式进行横断面调查。

重要结论: 护士人群的 IBS 患病率达 $17.4 \%$, 与健康对照组相比, IBS 患者精神心理评分升高 $(P<0.001)$, 且生活质量明显降低（77.18 \pm 21.93 vs. $88.44 \pm 11.89, P<0.001)$ 。饮酒、缺乏锻炼、精神心理异 常等因素可能和 IBS 患病有关。

关键词组：肠易激综合征（IBS）; 患病率; 精神心理; 生活质量 